\title{
Cosmology with Mimetic Matter
}

\author{
Alexander Vikman* \\ Ludwig-Maximilians-Universität München \\ E-mail: vikmandecern.ch
}

This talk will be mostly based on [2]. I will discuss cosmology of the recently introduced "mimetic" modification of general relativity [1]. This modification is manifestly Weyl-invariant and can mimic practically any cosmological époque $[2,3]$. In particular, this "mimetic" modification can naturally account for a part of dark matter [2,3]. It is also possible to unify such dark matter with dark energy as it was shown, in [3]. Finally one can construct an interesting single-field model for the early universe inflation. This model has parametrically suppressed gravity waves but does not seem to produce any non-Gaussianity, moreover the usual consistency relation for the single-field inflationary models gets modified.

References

[1] A. H. Chamseddine, V. Mukhanov, Mimetic Dark Matter, JHEP 1311 (2013) 135, arXiv: 1308.5410

[2] A. H. Chamseddine, V. Mukhanov, and A. Vikman, Cosmology with Mimetic Matter, JCAP 1406 (2014) 017, arXiv:1403.3961

[3] E. A. Lim, I. Sawicki, and A. Vikman, Dust of Dark Energy, JCAP 1005 (2010) 012, arXiv:1003.5751

Frontiers of Fundamental Physics 14 - FFP14,

15-18 July 2014

Aix Marseille University (AMU) Saint-Charles Campus, Marseille

${ }^{*}$ Speaker. 